\title{
VINCAMINA EM PACIENTES COM INSUFICIENCIA VASCULAR CEREBRAL
}

\author{
SÉrgio Pereira Novis * \\ MAFALDA MORETTO ** \\ Sheila B. Fenelon *** \\ Cláudro S. Barbosa *** \\ JANILDE DA GRAÇA TORRES ***
}

Os modernos conceitos fisiopatológicos da circulação encefálica vieram reformular a terapêutica das afecções vasculares cerebrais.

As experiências de Lassen ${ }^{5}$ determinando a existência da síndrome de perfusão de luxo, por perturbações da auto-regulação da circulação encefálica quando atingidos os vasos cerebrais por processo isquêmico, bem como a sindrome do roubo cerebral, afastaram como agentes medicamentosos úteis nessas afecções alguns vasodilatadores classicamente utilizados.

Em 1953, Schilter, citado por Burban ${ }^{2}$, descobriu o princípio ativo da "pequena pervanche" (Vincamina) cuja planta já era usada de modo empírico há centenas de anos, sendo reconhecidos seus efeitos sobre vertigens, cefaléia e conhecida sua ação sedativa, abrindo novos horizontes na terapêutica dessas condições mórbidas. A obtenção da vincamina em escala industrial se deve às pesquisas de Szasz, citado por Burban ${ }^{2}$.

Animados pelos resultados obtidos por diversos autores nacionais $1,6,7$ e estrangeiros com a utilização desse medicamento no tratamento da insuficiência vascular cerebral, resolvemos estudar seus efeitos em pacientes selecionados na Clínica Neurológica da Policlínica Geral do Rio de Janeiro.

A vincamina, sob o ponto de vista químico, é a metil-16-hidroxi-14-carbometil-pirido-cantine $(1,2,3$ - c, d). As experiências realizadas com esta substância mostraram que ela provoca aumento da irrigação cerebral e melhoria circulatória coronária, acelera a velocidade sangüínea nos capilares cerebrais, tem efeitos hipotensores discretos e não provoca hipotensão ortostática ${ }^{10,12}$. Efeitos colaterais, bem como toxicidade, não foram observados por qualquer dos pesquisadores $8,9,11$.

Espagno, Lazorthes e Arbous ${ }^{3}$, utilizando o método do protóxido de azoto para a determinação do fluxo sangüineo cerebral, confirmaram o aumento obtido com o emprego deste medicamento em pacientes sofredores de insu-

Trabalho da Clinica Neurológica da Policlínica Geral do Rio de Janeiro:

* Diretor; ** Psicóloga; *** Médicos Assistentes. 
ficiência circulatória cerebral intermitente e perturbações pós-traumáticas da consciência.

O registro eletrencefalográfico contínuo, após injeção intramuscular de vincamina mostra evidente melhora da morfologia do ritmo alfa ${ }^{4}$.

\section{MATERIAL E METODOS}

Selecionamos 20 pacientes (Tabela 1) que apresentassem sinais clínicos de insuficiência vascular cerebral e que ainda não estivessem sendo submetidos a qualquer terapêutica especifica. Suas idades variavam de 40 a 72 anos, sendo 9 homens e 11 mulheres. Todos foram submetidos a cuidadosa investigação clinfca constando de exame clínico e neurológico, eletrencefalograma, exames laboratoriais e testes psicométricos.

Os testes psicométricos foram planejados e aplicados como segue: a partir do estabelecimento do objetivo da pesquisa e das caracteristicas dos pacientes que a ela se submeteriam, foi elaborada uma bateria de testes que permitisse avaliar de maneira mais efetiva os fins pré-estabelecidos; levando-se em consideração que no polimorfo aspecto clínico da insuficiência vascular cerebral os distúrbios da memória são dos mais significativos, foi elaborada uma bateria visando a avaliar o rendimento dos doentes nesta área e nas que se relacionassem com o distúrbio acima mencionado. A bateria utilizada foi a seguinte: nivel mental; memória anterógrada; memória retrógrada (visual e auditiva); atenção concentrada; raciocínio abstrato; personalidade Rorschach.

Utilizamos o teste de personalidade a fim de avaliar a existência de possiveis interferências emocionais no rendimento dos testes empregados. Os doentes foram igualmente submetidos a entrevista com a psicóloga que lhes explicava a finalidade da pesquisa e, também, buscava evidenciar problemas na esfera familiar e social que pudessem interferir nos resultados. Apesar de todo este cuidado houve 5 pacientes (casos 3, 7, 13, 19 e 20) que não compareceram para a revisão final após - periodo da pesquisa, não sendo possível a obtencão de qualquer contacto ulterior com os mesmos. Como o caso 17 abandonou 0 tratamento, nas tabelas 2 e 3 figuram os resultados em 14 pacientes.

Os testes psicométricos colocavam os pacientes frente a séries de itens, problemas ou perguntas elaboradas de antemão, dos quais pudessem ser extraídos resultados totais ou parciais que permitissem comparar o desempenho dos doentes com o obtido em um grupo normativo. Os procedimentos para aplicação dos testes foram rigorosamente definidos a fim de assegurar a constância em sua administração. A obtenção dos resultados foi generalizada, procurando-se que estes resultados fossem tão independentes do juizo pessoal do examinador quanto possivel; realizou-se contando, controlando por meio de chaves ou consultando o manual no qual eram detalhadas as regras específicas para o cômputo. Com isto consegue-se que a pesquisa possa ser verificada por outros examinadores, que é o ideal das técnicas psicométricas, isto é, proporcionar resultados consistentes quando fossem repetidos após um certo periodo e também independentes tanto do lugar como da personalidade do examinador.

Iniciou-se então o trabalho a partír de uma mostragem aleatória constitúda pelos 20 pacientes selecionados. Estes enfermos, com nível de instrução primárío, ginasial e superior, distribufam-se sob o ponto de vista sócio-econômico nas classes baixa e média.

Depois que todos os 20 pacientes foram submetidos à testagem, iniciamos o emprego da vincamina *, na dose de $40 \mathrm{mg}$ nas 24 horas por via oral, em comprimidos de $10 \mathrm{mg}$ ingeridos dois pela manhã e dois à noite.

* Medicamento fornecido por Laboratórios Lepetit S.A. (Perval) 
Decorrido um prazo de 15 dias desde o inicio da medicação, foram os doentes novamente submetidos a exame clínico e neurológico, eletrencefalograma e aos mesmos testes aplicados para avaliação psicométrica.

\section{CASUISTICA}

Os dados referentes aos pacientes que se submeteram a esta pesquisa estão relacionados nas tabelas 1,2 e 3 que, mostrando de modo mais objetivo não só os informes iniclais como também os resultados obtidos com o emprego do medicamento, proporcionam uma visão mais rápida e realista do que pudemos verificar.

\begin{tabular}{|c|c|c|c|c|c|}
\hline Caso & Reg. & $\begin{array}{l}\text { Idade } \\
\text { Sexo } \\
\text { Cor }\end{array}$ & Quadro clinico & Resultado & \\
\hline 1 & 1089 & $\begin{array}{l}60 \\
\text { F } \\
\text { B }\end{array}$ & $\begin{array}{l}\text { Traumatismo craniencefálico há } \mathbf{8} \\
\text { anos. Hipomnèsia. }\end{array}$ & melhorado & \\
\hline 2 & 1436 & $\begin{array}{l}50 \\
\mathbf{F} \\
\mathbf{B}\end{array}$ & $\begin{array}{l}\text { Crises de perda de visão, vertigens, } \\
\text { hipomnésia. }\end{array}$ & melhorado & \\
\hline 3 & 1448 & $\begin{array}{l}61 \\
\text { F } \\
\text { B }\end{array}$ & $\begin{array}{l}\text { Vertigens, insônia, parestesias dimidio } \\
\text { D., crises dim. acuidade visual D., hi- } \\
\text { pomnésia. }\end{array}$ & $\begin{array}{l}\text { abandonou } \\
\text { pesquisa }\end{array}$ & a \\
\hline 4 & 1483 & $\begin{array}{l}60 \\
\mathbf{F} \\
\mathbf{B}\end{array}$ & $\begin{array}{l}\text { Vertigens, hipomnésia F.O. - grau II } \\
\text { W.K. }\end{array}$ & melhorado & \\
\hline 5 & 1494 & $\begin{array}{l}64 \\
\mathbf{F} \\
\mathbf{B}\end{array}$ & $\begin{array}{l}\text { Cefaléia, vertigens, parestesias, escoto- } \\
\text { mas, hipomnésia. }\end{array}$ & melhorado & \\
\hline 6 & 1510 & $\begin{array}{l}72 \\
\mathrm{M} \\
\mathrm{B}\end{array}$ & $\begin{array}{l}\text { Cefaléia, vertigens, perda da consciên- } \\
\text { cia há um mês, hipomnésia. }\end{array}$ & melhorado & \\
\hline 7 & 1514 & $\begin{array}{l}57 \\
\text { M } \\
\text { B }\end{array}$ & $\begin{array}{l}\text { Monoparesia braquial E., hipomnésia, } \\
\text { sinais piramid. MSE. }\end{array}$ & $\begin{array}{l}\text { abandonou } \\
\text { pesquisa }\end{array}$ & a \\
\hline 8 & 1546 & $\begin{array}{l}70 \\
\text { F } \\
\text { B }\end{array}$ & Vertigens, hipomnésia. & melhorado & \\
\hline 9 & 1553 & $\begin{array}{l}64 \\
\text { F } \\
\text { B }\end{array}$ & $\begin{array}{l}\text { Parestesia peribucal, dores em queima- } \\
\text { cão dimidio E., hipomnésia, infarto mio- } \\
\text { cárdio há } 5 \text { anos. }\end{array}$ & melhorado & \\
\hline 10 & 1555 & $\begin{array}{l}60 \\
\mathrm{M} \\
\mathrm{B}\end{array}$ & Vertigens, hipomnésia. & inalterado & \\
\hline 11 & 1596 & $\begin{array}{l}\mathbf{5 1} \\
\mathbf{F} \\
\mathbf{B}\end{array}$ & Hipomnésia F.O. grau II W.K. & melhorado & \\
\hline 12 & 1661 & $\begin{array}{l}\mathbf{5 7} \\
\mathrm{F} \\
\mathrm{B}\end{array}$ & $\begin{array}{l}\text { Sindrome confusional, crises de diplo- } \\
\text { pia, hipomnésia. }\end{array}$ & melhorado & \\
\hline 13 & 1675 & $\begin{array}{l}55 \\
\text { F } \\
\text { B }\end{array}$ & $\begin{array}{l}\text { Insônia, hipomnésia. } \\
\qquad \text { continua }\end{array}$ & $\begin{array}{l}\text { abandonou } \\
\text { pesquisa }\end{array}$ & $\mathbf{a}$ \\
\hline
\end{tabular}




\begin{tabular}{|c|c|c|c|c|}
\hline Caso & Reg. & $\begin{array}{l}\text { Idade } \\
\text { Sexo } \\
\text { Cor }\end{array}$ & Quadro clínico & Resultado \\
\hline 14 & 1750 & $\begin{array}{l}50 \\
\mathrm{M} \\
\mathrm{B}\end{array}$ & $\begin{array}{l}\text { Nucalgia, dim. acuidade visual, derrea- } \\
\text { mento membros inferiores, hipomnésia. }\end{array}$ & inalterado \\
\hline 15 & 1752 & $\begin{array}{l}63 \\
\text { M } \\
\text { B }\end{array}$ & $\begin{array}{l}\text { Crises de perda da acuidade visual, } \\
\text { hipomnésia, pulso carotideo D. }\end{array}$ & inalterado \\
\hline 16 & 1758 & $\begin{array}{l}49 \\
\mathrm{M} \\
\mathrm{B}\end{array}$ & Vertigens, hipomnésia. & inalterado \\
\hline 17 & 2005 & $\begin{array}{l}57 \\
\mathrm{M} \\
\mathrm{B}\end{array}$ & $\begin{array}{l}\text { Insônia, bradipsiquismo, hipomnésia, } \\
\text { crises perda consciência F.O. grau II } \\
\text { W.K. }\end{array}$ & $\begin{array}{l}\text { abandonou } \\
\text { tratamento }\end{array}$ \\
\hline 18 & 2124 & $\begin{array}{l}\mathbf{5 5} \\
\mathbf{M} \\
\mathbf{B}\end{array}$ & Vertigens, hipoacusia AO, hipomnésia. & melhorado \\
\hline 19 & 2790 & $\begin{array}{l}40 \\
\text { M } \\
\text { B }\end{array}$ & $\begin{array}{l}\text { Vertigens, claudicação intermitente, hi- } \\
\text { pomnésia. }\end{array}$ & $\begin{array}{l}\text { abandonou } \\
\text { pesquisa }\end{array}$ \\
\hline 20 & 1610 & $\begin{array}{l}60 \\
\text { F } \\
\text { B }\end{array}$ & Acidente vasc. cerebral, hemiparesia $\mathbf{E}$. & $\begin{array}{l}\text { abandonou a } \\
\text { pesquisa }\end{array}$ \\
\hline
\end{tabular}

Tabela 1 - Dados de identificação dos 20 pacientes, respectivo quadro clínico $e$ resultados aerais apnos o tratamento com a vincamina.

\begin{tabular}{|c|c|c|c|c|c|c|c|}
\hline \multirow[t]{2}{*}{ Caso } & \multirow[t]{2}{*}{ Escol. } & \multicolumn{2}{|c|}{ Nivel mental } & \multicolumn{2}{|c|}{ Raciocinio abstrato } & \multicolumn{2}{|c|}{ Atencão concentrada } \\
\hline & & antes & depois & antes & depois & antes & depois \\
\hline 1 & Gin. & $P_{25}$ & $P_{50}$ & 8 & 10 & Infrad. & Médio \\
\hline 2 & Prim. & $P_{40}$ & $P_{25_{M I}}$ & 5 & 7 & Médio & Médio \\
\hline 4 & Prim. & $P_{25_{M I}}$ & $\mathrm{P}_{25_{\mathrm{MI}}}$ & 6 & 4 & Infrad. & Infrad. \\
\hline 5 & Prim. & $P_{25}$ & $P_{25}$ & 8 & 7 & Médio & Infer. \\
\hline 6 & Sup. & $P_{50 M}$ & $P_{10_{I}}$ & 9 & 10 & Infrad. & Infrad. \\
\hline 8 & Sec. & $\mathrm{P}_{50_{\mathrm{M}}}$ & $P_{25}$ & 9 & 6 & Infrad. & Infrad. \\
\hline 9 & Prim. & $P_{25 \mathrm{MI}}$ & $P_{50}$ & 6 & 7 & Intraũ. & Infrad. \\
\hline 10 & Gin. & $\mathbf{P}_{50}$ & $P_{50 M}$ & 9 & 12 & Infrad. & Médio \\
\hline 11 & Prim. & $P_{5_{I}}$ & $P_{5_{I}}$ & 10 & 10 & Infrad. & $\begin{array}{l}\text { Médio } \\
\text { Infer. }\end{array}$ \\
\hline 12 & Prim. & $\mathrm{P}_{50} \mathrm{M}$ & $P_{50}$ & 0 & 5 & $\begin{array}{l}\text { Médio } \\
\text { Infer. }\end{array}$ & $\begin{array}{l}\text { Médio } \\
\text { Infer. }\end{array}$ \\
\hline 14 & Prim. & $P_{25}$ & $P_{50}{ }_{M I}$ & 6 & 10 & Infrad. & Infrad. \\
\hline 15 & Gin. & $P_{25}$ & $\mathbf{P}_{25_{\mathrm{MI}}}$ & 8 & 10 & Infrad. & $\begin{array}{l}\text { Médio } \\
\text { Infer. }\end{array}$ \\
\hline 16 & Gin. & $P_{25}$ & $\mathbf{P}_{25_{\mathrm{MI}}}$ & 8 & 9 & Infrad. & Infrad. \\
\hline 18 & Gin. & $\mathrm{P}_{75_{\mathrm{MS}}}$ & $\mathrm{P}_{75_{\mathrm{MS}}}$ & 8 & 15 & Médio & Infer. \\
\hline
\end{tabular}

Tabela 2 - Resultados obtidos com os testes psicométricos de nivel mental, raciocínio abstrato $e$ atenção concentrada antes $e$ depois do tratamento com vincamina em 14 pacientes: Escol. = escolaridade; Gin. = ginasial; Prim. = primário; Sec. $\approx$ secundário. 


\begin{tabular}{|c|c|c|c|c|c|c|}
\hline \multirow[t]{2}{*}{ Caso } & \multicolumn{2}{|c|}{ Mem. anterógrada } & \multicolumn{2}{|c|}{ Mem. visual } & \multicolumn{2}{|c|}{ Mem. auditiva } \\
\hline & antes & depois & antes & depois & antes & depois \\
\hline 1 & Infer. & Infer. & $\begin{array}{l}\text { Médio } \\
\text { Super. }\end{array}$ & Superior & Infrad. & $\begin{array}{l}\text { Médio } \\
\text { Infer. }\end{array}$ \\
\hline 2 & Infer. & Infer. & Médio & Superior & Infrad. & Superior \\
\hline 4 & Infer. & $\begin{array}{l}\text { Médio } \\
\text { Infer. }\end{array}$ & Infrad. & $\begin{array}{l}\text { Médio } \\
\text { Infer. }\end{array}$ & Infrad. & Infrad. \\
\hline 5 & Infer. & Infer. & Infer. & $\begin{array}{l}\text { Médio } \\
\text { Superior }\end{array}$ & Infrad. & Infrad. \\
\hline 6 & Infer. & Infer. & Infrad. & Infrad. & Infrad. & Infrad. \\
\hline 8 & Infer. & Infer. & Infrad. & Infrad. & Infrad. & Infrad. \\
\hline 9 & Infer. & Infer. & Infrad. & $\begin{array}{l}\text { Médio } \\
\text { Infer. }\end{array}$ & Infer. & Infer. \\
\hline 10 & Infer. & Infer. & Infer. & Médio & Infrad. & Infrad. \\
\hline 11 & Infer. & Infer. & Infer. & Médio & Infrad. & $\begin{array}{l}\text { Médio } \\
\text { Infer. }\end{array}$ \\
\hline 12 & Infer. & $\begin{array}{l}\text { Médio } \\
\text { Infer: }\end{array}$ & Superior & Superior & Infer. & $\begin{array}{l}\text { Médio } \\
\text { Infer. }\end{array}$ \\
\hline 14 & Infer. & Infer. & Infrad. & Infrad. & Infer. & Infer. \\
\hline 15 & Infer. & Infer. & Infer. & Médio & Infrad. & $\begin{array}{l}\text { Médio } \\
\text { Infer. }\end{array}$ \\
\hline 16 & Infer. & Infer. & Infrad. & Superior & Infrad. & $\begin{array}{l}\text { Médio } \\
\text { Infer. }\end{array}$ \\
\hline 18 & Infer. & $\begin{array}{l}\text { Médio } \\
\text { Infer. }\end{array}$ & Médio & Médio & Infrad. & Superior \\
\hline
\end{tabular}

Tabela 3 - Resultados obtidos nos testes de memória anterógrada e retrógrada (visual e auditiva) antes e depois do tratamento com vincamina em 14 pacientes.

\section{RESULTADOS}

Da análise de nossa casuistica, pudemos observar ser a víncamina uma substância eficaz no tratamento das polimorfas manifestações clínicas da insuficiência vascular cerebral. De fato, verificamos que dos 14 pacientes submetidos ao tratamento durante 150 dias e nos quais foi feita cuidadosa revisão final, 10 apresentaram melhoras clínicas (65\% dos casos). Em nenhum dos pacientes foi verificada intolerância medicamentosa, bem como não se registrou hipotensão ortostática.

A sintomatologia predominante era a hipomnésia, presente na totalidade dos casos do tipo classicamente descrito na arteriosclerose cerebral, isto é, com malor deficiência da memória anterógrada. Foram observadas também manifestações vertiginosas, cefaléia, insônia, parestesias, paresias e paralisias, crises de diminuição da aculdade visual, fenômenos estes observados habitualmente nas insuficiências vasculares cerebrais seja no território carotídeo e/ou no vértebro-basilar. Estas manifestações, conforme acima assinalamos, regrediram em $65 \%$ dos casos, sendo que alguns se tornaram assintomáticos.

\section{TESTES PSICOMETRICOS}

Vários autores têm feito referência à eficácia da vincamina no combate a distúrbios da memória. Entre eles salienta-se, em nosso meio, Oliveira ${ }^{7}$ 
que confirmou este fato. Pelo que se pode depreender da análise das tabelas referentes aos testes psicométricos, nossos pacientes mostraram melhoras, em graus diversos, nos diferentes testes objetivos visando a analisar esta importante função psíquica.

Em resumo foi o seguinte o que observamos: quanto ao nivel mental melhoraram 21,4\% dos pacientes, permaneceram estacionários $57,1 \%$ e pioraram 21,4\%. Memória anterógrada: melhoraram 21,4\%, permaneceram estacionários 78,5\%. Memória retrógrada: visual, melhoraram 57,1\% e permaneceram estacionários 43,5\%; auditiva, melhoraram $50,0 \%$ e permaneceram estacionários 50,0\%. Atenção concentrada: melhoraram 28,5\%, permaneceram estacionários $57,1 \%$, pioraram 14,2\%. Raciocínio abstrato (Wechsler): melhoraram $71,4 \%$, permaneceram estacionários $7,1 \%$, pioraram $21,4 \%$.

Os pacientes foram igualmente submetidos ao teste de Rorschach que, verificando a situação mental do examinado, permite dirimir dúvidas quanto à correta interpretação dos sintomas clínicos apresentados, sobretudo os referentes a aspectos mais complexos como o da avaliação da memória. Curiosamente não encontramos nos trabalhos compulsados este cuidado técnico que nos parece sobremodo importante para a valorização dos resultados obtidos.

Na primeira etapa da testagem, 6 pacientes dos 14 que se submeteram à totalidade dos testes psicométricos na avaliação final, apresentaram protocolo normal para a idade; os 8 restantes tiveram protocolo anormal. Nestes últimos foram observados os seguintes sintomas: do ponto de vista intelectual, grande tendência para tirar conclusões deformadas e conseqüentes atitudes de choque frente à realidade. Os conceitos formais apresentaram-se com acentuada imprecisão, evidenciando, na maioria das vezes, um tipo de percepção predominantemente global, com preocupação para com o concreto das coisas. Estes achados são os que habitualmente se encontram em pacientes com arteriosclerose cerebral. No que concerne ao controle do pensamento lógico e atenção foi evidenciado grande prejuízo.

$\mathrm{Na}$ esfera emocional alguns pacientes mostraram baixa susceptibilidade aos estímulos emocionais e outros, em oposição, certa explosividade em suas emoções. De modo geral, manifestaram regressão da afetividade, voltando a apresentar a labilidade das primeiras fases da curva etária. Observamos também, freqüentemente, ansiedade, insegurança, pensamentos esteriotipados e traços de oposição. Do ponto de vista social, a capacidade de relacionamento estava bastante prejudicada, uma vez que não participavam do pensamento coletivo ou o faziam passivamente.

Na segunda etapa da testagem, após o tratamento por 150 dias, 11 pacientes dos 14 examinados, evidenciaram significativas melhoras. Um mostrou-se inalterado e dois casos revelaram protocolos com piora nos resultados.

As melhoras mais significativas foram encontradas na capacidade de controle do pensamento lógico, na atenção e na possibilidade de tirar conclusões acertadas. Houve significativa redução da ansiedade e impulsividade. 
Em quase todos os casos foi observado o desaparecimento de traços oposicionistas. Notamos, igualmente, diminuição na estereotipia do pensamento. $\mathrm{Na}$ maioria dos casos passaram os pacientes a agir de maneira menos construtiva, o que permitiu reação aos estímulos mais segura e menos ansiosa, tornando-e, inclusive, menos passivos em relação aos grupos.

Acreditamos que a melhora observada do ponto de vista psíquico em $\mathbf{1 1}$ dos 14 pacientes examinados se deva sobretudo a: a) ação eficaz do medicamento, melhorando o quadro clínico e conseqüentemente gerando maior segurança ao enfermo; b) correto relacionamento entre os doentes e a equipe de trabalho, salientando-se, no entanto, não terem sido submetidos a práticas psicoterápicas.

Alguns autores já haviam assinalado o fato de que a vincamina tem ação sobre o traçado eletrencefalográfico, sobretudo regularizando o ritmo alfa 4 Efetivamente, dos 14 pacientes que apresentaram anormalidade eletrencefalográfica antes do tratamento verificamos, em 7, melhora do traçado.

\section{RESUMO}

Foram estudados, em 20 casos de pacientes com insuficiência vascular cerebral, os efeitos do tratamento com a vincamina. Os doentes foram previamente submetidos a exames neurológico, eletrencefalográfico, laboratoriais, psicométricos (teste de memória, teste de capacidade intelectiva, teste de atenção concentrada, raciocínio abstrato e de personalidade). Os pacientes foram medicados com vincamina na dose de $40 \mathrm{mg}$ nas 24 horas durante o período aproximado de 150 dias, após o que foram novamente submetidos à análise idêntica. Os resultados obtidos, apresentados em tabelas, revelam que, em razoável percentual, foi a vincamina medicamento útil no controle das manifestações clínicas da insuficiência vascular cerebral, sendo evidenciada melhora nos indices de déficit de memória retrógrada e anterógrada observados nos testes objetivos. Houve também melhora no padrão eletrencefalográfico de alguns pacientes.

\section{SUMMARY}

\section{Effects of vincamine in patients with cerebrovascular insufficiency}

The effects of the treatment with vincamine in 20 patients with cerebral vascular insufficiency are reported. The patients were previously submitted to the following tests: neurologic examination, electroencephalography, laboratory tests, psychometry (memory, intellective capability, concentrated attention, abstract reasoning and personality tests). Patients were medicated with vincamin in a $40 \mathrm{mg}$ dose within 24 hours during approximately a 150 days period, after which were again submitted to an identical analysis. The results obtained revealed that in a reasonable percentage of the cases vincamin is an useful medication in controlling the clinical manifestations 
of the cerebral vascular insufficiency, an improvement being demonstrated in the retrograde and anterograde deficit rate observed in objective tests. An improvement was also noticed in the electroencephalograpric pattern of a few patients.

\section{REFERENCIAS}

1. ASPesi, N.; Balaguez, H. M.; hausen, S. R. \& PRates, F. P. C. Estudos clínicos da vincamina nas afeccões cerebro-vasculares agudas. Folha Médica 65:73, 1972.

2. BURBAN, J. - Contribution a l'étude de l'action de la vincamine dans l'insuffisance circulatoire cérebrale. These pour le Doctorat en Medecine, Université de Clermont, 1968.

3. ESPAGNO, J.; LAZORTHES, Y. \& ARBUS, L. - Techniques d'étude hemodynamique et metabolique d'un medicament a visée vasculaire cérebrale. Therapeutique Bichat, 1969.

4. FOLDI, M. \& OBAL, F. - L'effet de la vincamine sur la circulation capillaire du cerveau et sur l'E.E.G. Therapia Hungarica 13:95, 1965.

5. LASSEN, N. A. - The luxury perfusion syndrome and its possible relation to acute metabolic acidosis localized within the brain. Lancet $2: 1113,1964$.

6. LIMA COSTA, A. - Ensaio clínico com nova substância vaso-ativa em nível cerebral. Folha Médica 65:1047, 1972.

7. OLIVEIRA, J. M. - Estudos realizados com a vincamina nos testes de memória. Folha Médica 65:51, 1952.

8. PECKER, J. \& SCARABIN, J. M. - Experimentation clinique de la Pervincamine en neuro-chirurgie. Quest. Med. 24:1007, 1971.

9. RAVINA, A. - Une indication therapeutique nouvelle des alcaloides de la pervenche. Prèsse Médicale 74:525, 1966.

10. SOLTI, F.; ISKUM, M.; PETER, A.; REV, J.; HERMANN, R. \& FOLDESY, K. - Examens sur des êtres humains au sujet de l'effet exercé par le Devincan sur la circulation cérèbrale, la pression veineuse cérébrale et la consommation d'oxygene du cerveau. Cor et Nasa, 16:138, 1964.

11. SZABOR, A. - Spasmolytic treatment os cerebrovascular diseases. Therapia Hungarica 18:63, 1970.

12. WAROT, P. \& CARON, J. C. - Les traitment des accidents ischemiques cerebraux par la pervincamine. Lille Medical 16:614-618, 1971.

Clinica Neurológica da Policlínica Geral do Rio de Janeiro - Av. Nilo Pecanha $38,6^{\circ}$ andar - 20000 Rio de Janeiro, GB - Brasil. 